\title{
The establishment and use of an in vivo animal model for cervical intra-epithelial neoplasia.
}

\begin{abstract}
Cervical cancer is the second most common cancer of female reproductive tracts. In developing countries, cervical carcinoma is the leading cause of cancer fatality in women Despite attempts to lower the fatality rate, very few in vivo models are in place to investigate this cancer. We therefore are able to develop an in vivo animal model that is suitabie to conduct such study. In our attempt to secure an in vivo animal model for cervical cancer, the carcinogenic property of diethylstilboestrol (DES) was exploited to establish a model for Cervical Intruepithelial Neoplasia or carcinoma (CIN). Female-Balb/C mice were injected with several dosages of DES (i.p) during pregnancy at day 13-18. Female offspring were reared and sacrificed at age of 48-54 days and the cervix tissues taken for histological evaluation using $\mathrm{H}$ and $\mathrm{E}$. The progression of the cancer and hence, disease state is monitored by measuring serum IL-6 using an ELISA kit. Proliferative cell nuclear antigen (PCNA) expressions were studied by implying immunohistochemical techniques. All parameters with regards to CIN were compared to a control group of treating the cancer using a used drug, cisplatin, used preferentially to treat cervical cancer in humans. The results of this study revealed that a significant difference in serum IL-6 concentration between DES-treated group and control groups $(\mathrm{p}<.05)$. CIN histological related lesions was noticed to be prominently dominant in DES-treated animals whilst these lesions were absent in control groups. In addition to that PCNA index in DES-treated animal was found to be a significant different compared to control group. The above findings indicate that DES could be utilized and further exploited as cervical carcinogenesis initiator in animal models to screen and study new potential anti-cervical cancer compounds in vivo.
\end{abstract}

Keyword: Carcinogenesis; Cervical cancer; CIN in vivo models; Cisplatin; DES 